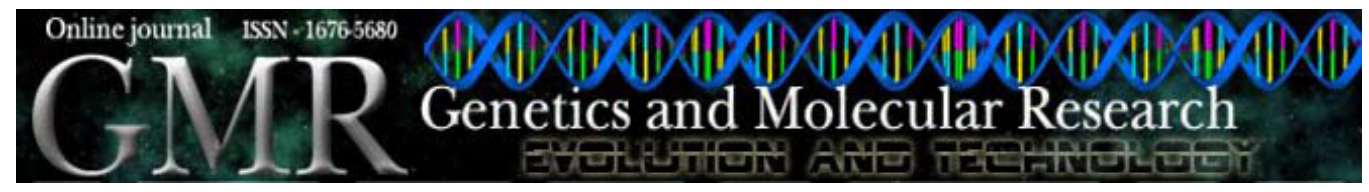

\title{
Genetic variation of Kaempferia (Zingiberaceae) in Thailand based on chloroplast DNA (psbA-trnH and petA-psbJ) sequences
}

\author{
J. Techaprasan ${ }^{1}$, S. Klinbunga ${ }^{1}$, C. Ngamriabsakul $^{2}$ and T. Jenjittikul ${ }^{3}$ \\ ${ }^{1}$ National Center for Genetic Engineering and Biotechnology, Klong Luang, \\ Pathum Thani, Thailand \\ ${ }^{2}$ School of Science, Walailak University, Nakhon Si Thammarat, Thailand \\ ${ }^{3}$ Department of Plant Science, Faculty of Science, Mahidol University, \\ Bangkok, Thailand
}

Corresponding author: J. Techaprasan

E-mail: jiranan@biotec.or.th

Genet. Mol. Res. 9 (4): 1957-1973 (2010)

Received May 9, 2010

Accepted July 18, 2010

Published October 5, 2010

DOI 10.4238/vol9-4gmr873

ABSTRACT. Genetic variation and species authentication of 71
Kaempferia accessions (representing 15 recognized, six new, and four
unidentified species) found indigenously in Thailand were examined
by determining chloroplast $p s b A$-trn $H$ and partial petA-psbJ spacer
sequences. Ten closely related species (Boesenbergia rotunda,
Gagnepainia godefroyi, G. thoreliana, Globba substrigosa, Smithatris
myanmarensis, S. supraneanae, Scaphochlamys biloba, S. minutiflora,
S. rubescens, and Stahlianthus sp) were also included. After sequence
alignments, 1010 and 865 bp in length were obtained for the respective
chloroplast DNA sequences. Intraspecific sequence variation was
not observed in Kaempferia candida, K. angustifolia, K. laotica, $K$.
galanga, K. pardi sp nov., K. bambusetorum sp nov., K. albomaculata
sp nov., K. minuta sp nov., Kaempferia sp nov. 1 , and $G$. thoreliana, for
which more than one specimen was available. In contrast, intraspecific
sequence polymorphisms were observed in various populations of $K$.
fallax, K. filifolia, K. elegans, K. pulchra, K. rotunda, K. marginata, $K$.
parviflora, K. larsenii, K. roscoeana, K. siamensis, and G. godefroyi. 
A strict consensus tree based on combined $p s b A$-trnH and partial petA$p s b J$ sequences revealed four major groups of Kaempferia species. We suggest that the genus Kaempferia is a polyphyletic group, as $K$. candida was distantly related and did not group with other Kaempferia species. Polymorphic sites and indels of $p s b A-t r n H$ and petA-psbJ can be used as DNA barcodes for species diagnosis of most Kaempferia and outgroup species. Nuclear DNA polymorphism should be examined to determine if there has been interspecific hybridization and chloroplast DNA introgression in these taxa.

Key words: Kaempferia; Chloroplast DNA; psbA-trnH; petA-psbJ; DNA barcode

\section{INTRODUCTION}

Kaempferia (Zingiberaceae) comprises about 60 species geographically distributed from India to Southeast Asia, where Thailand appears to be the richest biodiversity region with more than 20 extant species (Sirirugsa, 1992; Larsen and Saksuwan Larsen, 2006; Jenjittikul T and Larsen K, unpublished results). In Thailand, several Kaempferia species (i.e., K. grandifolia, K. galanga, K. marginata, K. elegans, and K. roscoeana) are well known for their ethnomedical uses by local people (Saensouk and Jenjittikul, 2001; Chuakul, 2003). Kaempferia parviflora (Krachai Dum) is famous as a health-promoting herb and is also used in several treatments such as dysentery, impotence, constriction, colic disorders, gastritis, etc. (Yenjai et al., 2003b, 2004).

Effective bioactive compounds have been isolated from several Kaempferia species. For instance, flavones (5-hydroxy-7-methoxyflavone and 5,7-dimethoxyflavone) from K. parviflora inhibited viral protease (Sookkongwaree et al., 2006). In addition, flavonoids (5,7,4'-trimethoxyflavone and 5,7,3',4'-tetramethoxyflavone) from $K$. parviflora and diterpenes (1,2,11-trihydroxypimara-8(14),15-diene and 1,11-dihydroxypimara-8(14),15-diene) from $K$. marginata exhibited anti-malarial activity against Plasmodium falciparum (Yenjai et al., 2004; Thongnest et al., 2005). Flavonoids (3,5,7,4'-tetramethoxyflavone and 5,7,4'-trimethoxyflavone) from $K$. parviflora and ethyl $p$-methoxycinnamate from $K$. galanga exhibited antimycobial activity against Mycobacterium tuberculosis and Candida albicans (Yenjai et al., 2003a,b, 2004). The ethanol extract of $K$. galanga exhibited anti-tumor promoter activity (Vimala et al., 1999). Therefore, plants in this genus are valuable sources of various bioactive compounds. In addition, Kaempferia are edible and valuable as ornamental plants and also used in cosmetic and perfume manufacturing (Ibrahim, 1999; Jenjittikul and Larsen, 2000; Saensouk and Jenjittikul, 2001).

Taxonomic identification of Kaempferia is difficult owing to the morphological similarity of vegetative parts among species and other genera in Zingiberaceae, such as Boesenbergia, Cornukaempferia, Curcuma, and Scaphochlamys. Without the floral parts, taxonomic identification to the species level is difficult. In addition, intraspecific variation causes more complicated problems in the classical taxonomy of this genus. Kaempferia angustifolia, for example, displays variations from narrow to broad leaves. The flowering season of Kaempferia is short, and inflorescences of some species (e.g., K. candida, K. grandifolia and $K$. rotunda) appear before leafy shoots and last only 1-2 weeks. In Thailand, most Kaempferia species are dormant during November to early May. Moreover, new species of Kaempferia have 
been increasingly discovered. At least four new Kaempferia species found in Thailand have been recently discovered (Jenjittikul T and Larsen K, unpublished results). Cytological studies of 12 Kaempferia species in Thailand were reported and varied enormously in the number of chromosomes $(2 \mathrm{n}=22,24,33,40,44$, and 55). Different ploidy levels were observed, e.g., in $K$. rotunda $(2 n=22$ or 33; Soontornchainaksaeng, 2005). The use of only vegetative morphological characters in the absence of reproductive parts and chromosome numbers is a major drawback for systematic and phylogenetic analyses and for authenticating morphologically similar Kaempferia species where no complete monograph is available at present (Saensouk and Jenjittikul, 2001).

Loesener (1930) taxonomically allocated 33 Kaempferia species into 5 subgenera. Of which, 9 species were found in Thailand and classified as members of subgenera Soncorus Horan. (i.e., $K$. galanga, $K$. roscoeana, $K$. glauca, $K$. pulchra, $K$. laotica, $K$. elegans, and $K$. angustifolia) and Protanthium Horan., including only precocious flowering species (i.e., $K$. candida and $K$. rotunda). However, more than 20 extant species have been recognized in Thailand to date.

Molecular systematics inferred from polymorphism of chloroplast (e.g., matK, trnK, trnL-trnF, and $p s b A$-trnH) and nuclear (i.e., internal transcribed spacer, ITS) DNA sequences have gained acceptance in resolving taxonomic problems that have arisen from the traditional classification. Polymorphic DNA sequences of chloroplast trnH-psbA intergenic spacer and nuclear ribosomal ITS have shown the potential to be used as DNA barcodes for species identification in biodiversity studies of 99 plant species, representing 80 genera from 53 families (Kress et al., 2005b). In Zingiberaceae, the polymorphism of chloroplast matK gene and nuclear ITS sequences was used to examine the molecular phylogeny of Alpinia (Kress et al., 2005a). Nucleotide sequence polymorphism and indels of $p s b A-\operatorname{trn} H$ and petA-psbJ spacers could be unambiguously applied as a molecular taxonomic key to authenticate 15 Boesenbergia species indigenous to Thailand. These data also supported further differentiation of $B$. bambusetorum from B. longiflora as a newly recognized Boesenbergia species (Techaprasan et al., 2006).

The genus Kaempferia is under revision with new species continually discovered (Larsen and Saksuwan Larsen, 2006). Little information of its phylogenetic history and molecular data inferred from chloroplast and/or nuclear sequences of Kaempferia has been reported. Recently, the phylogeny of Zingiberaceae was reported based on ITS and matK sequences, and Kaempferia is recognized as a monophyletic group (Kress et al., 2002). Nevertheless, only ITS sequences of 2 Kaempferia accessions and matK sequences of 4 Kaempferia taxa were included in the analyses. In this study, therefore, we applied a molecular approach in assessing the molecular systematics of Kaempferia found to be indigenous to Thailand using maternally inherited choloplast $p s b A-t r n H$ and petA-psbJ. The phylogeny and sequence polymorphism for systematics and species identification of various Kaempferia are reported for the first time in this genus.

\section{MATERIAL AND METHODS}

\section{Plant samples}

Seventy-one accessions of Kaempferia, representing 15 recognized, 6 new, and 4 unidentified Kaempferia species were collected throughout Thailand. Fourteen accessions, representing 10 closely related Zingiberaceae species (Boesenbergia rotunda, Gagnepainia godefroyi, G. thoreliana, Globba substrigosa, Scaphochlamys biloba, S. minutiflora, S. rubescens, Smithatris myanmarensis, $S$. supraneanae, and Stahlianthus $\mathrm{sp}$ ) were included as outgroups (Table 1). 


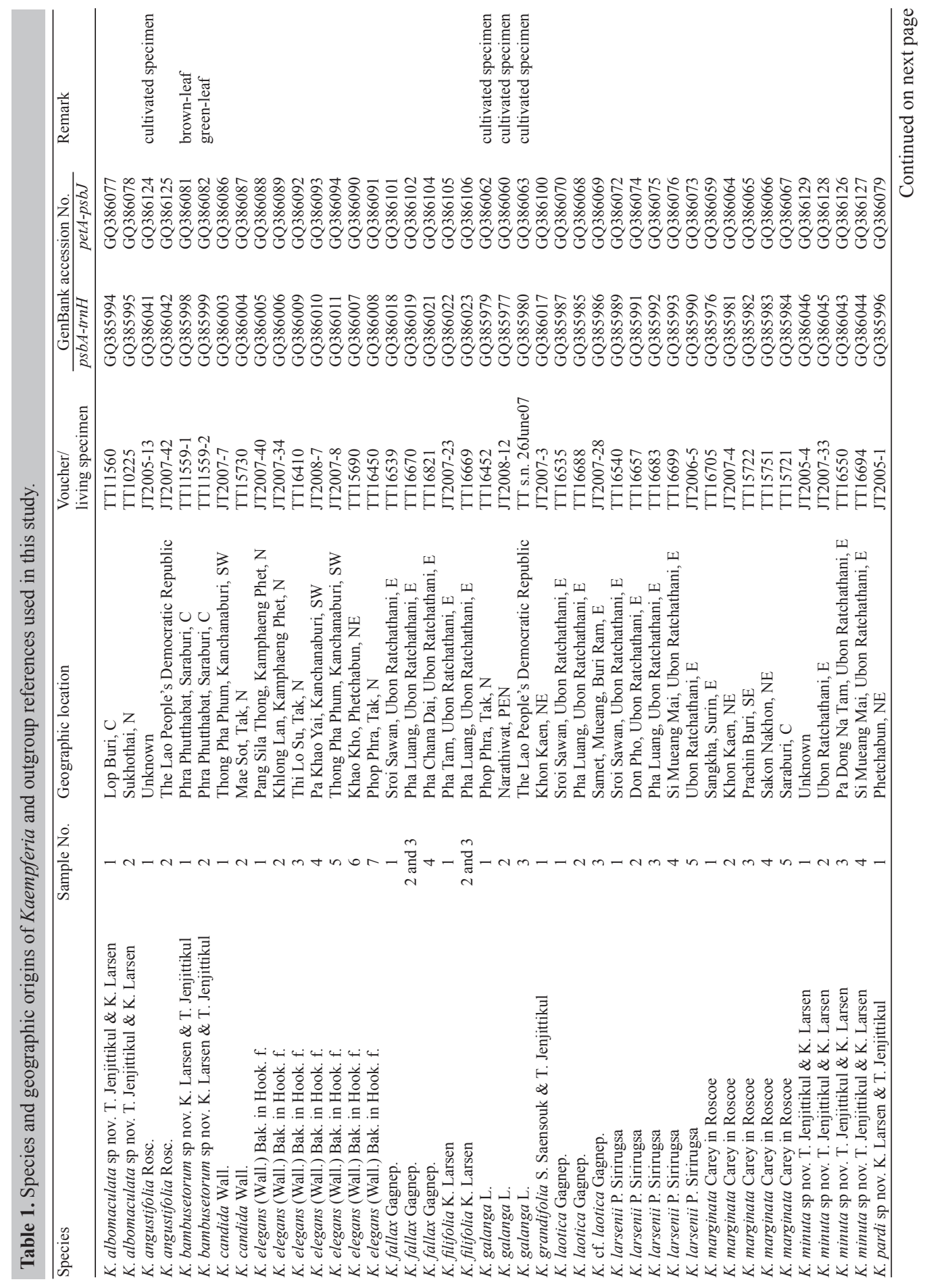




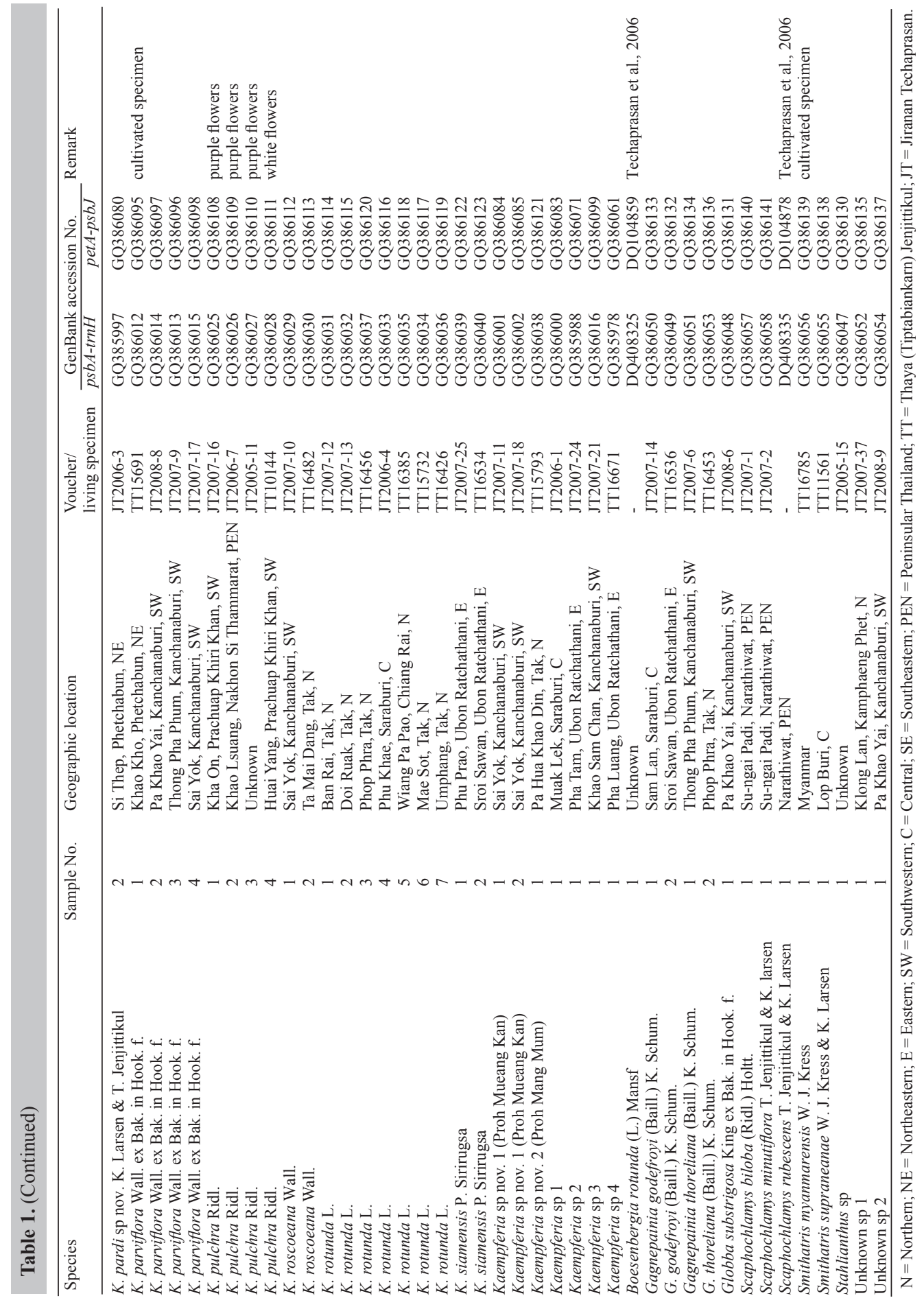


A map of Thailand illustrating sampling locations for replicate specimens of each Kaempferia species is shown in Figure 1. Voucher specimens were deposited at Suan Luang Rama IX herbarium, Thailand. The external morphology of some Kaempferia species in this study is shown in Figure 2.

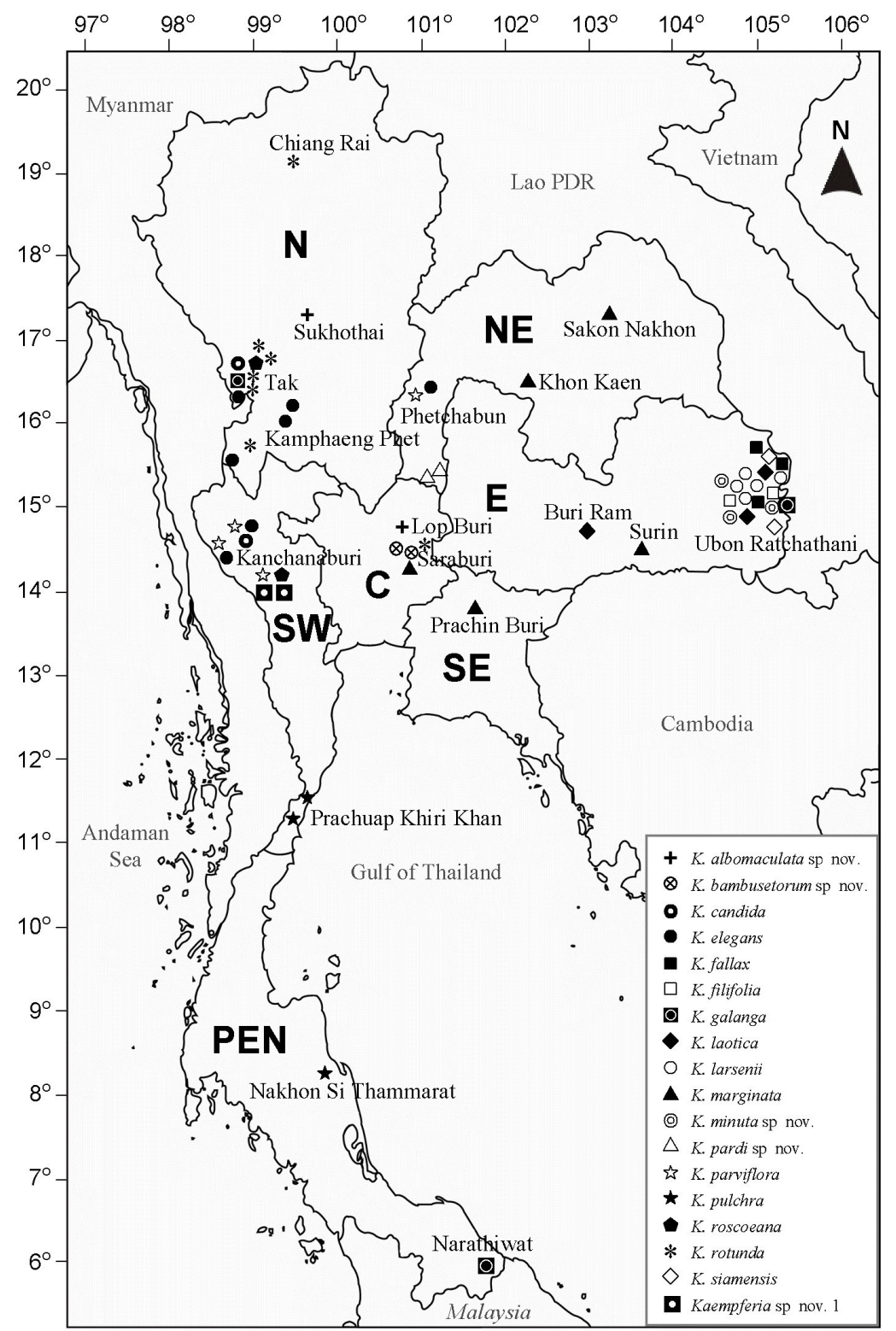

Figure 1. Map of Thailand showing multiple sample sites of various Kaempferia species. Solid lines indicate boundaries between floristic regions of Thailand. $\mathrm{N}=$ Northern; $\mathrm{NE}=$ Northeastern; $\mathrm{E}=$ Eastern; $\mathrm{SW}=$ Southwestern; $\mathrm{C}=$ Central; $\mathrm{SE}=$ Southeastern; $\mathrm{PEN}=$ Peninsular Thailand. 


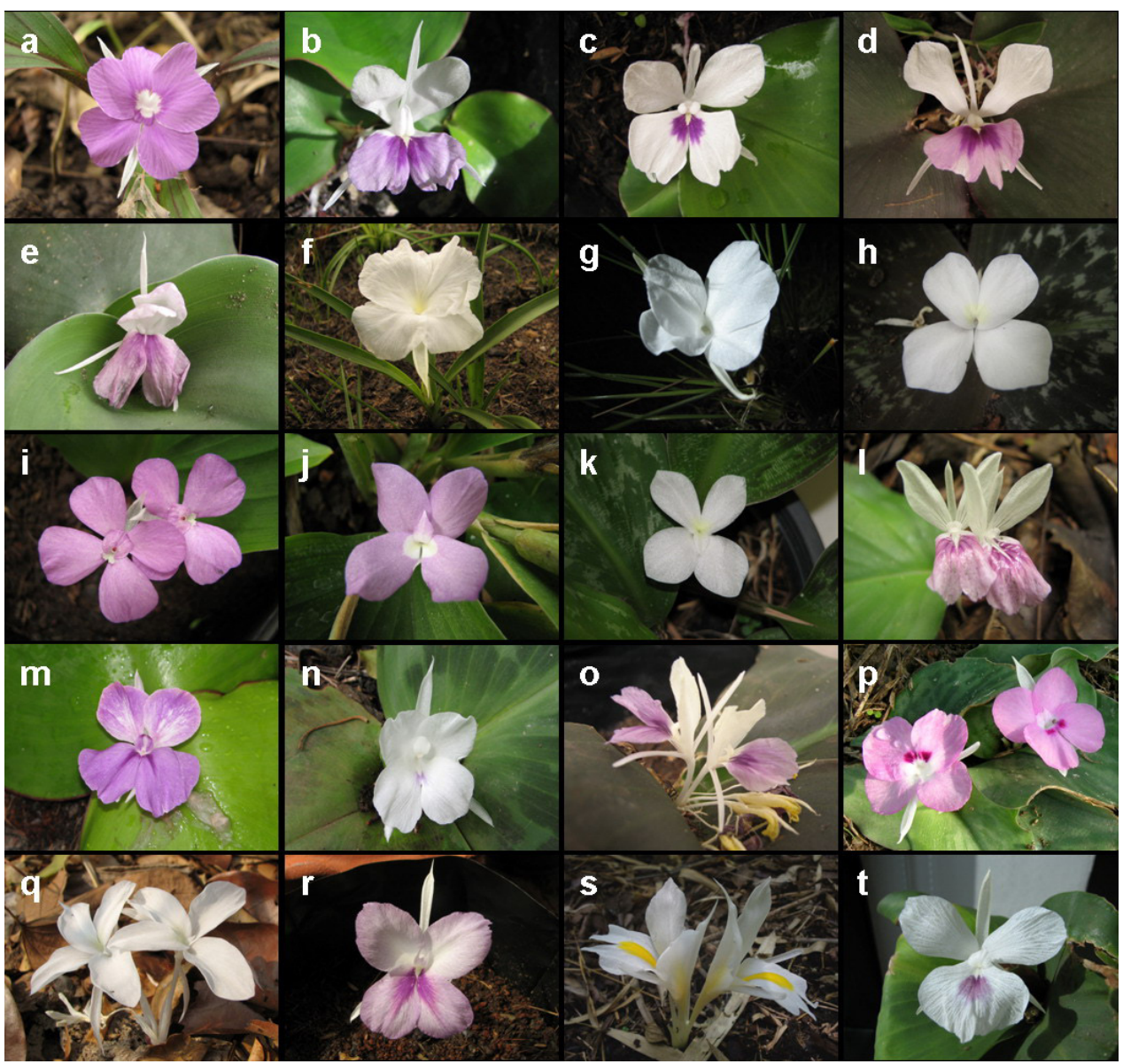

Figure 2. External morphology of some Kaempferia species. $K$. larsenii (a), K. laotica (b), $K$. galanga (c), $K$. marginata (d), K. siamensis (e), K. fallax (f), K. filifolia (g), K. roscoeana (h), K. elegans (i), K. pulchra (purple flower, j), K. pulchra (white flower, k), K. parviflora (1), K. albomaculata sp nov. (m), K. pardi sp nov. (n), K. bambusetorum sp nov. (o), K. minuta sp nov. (p), K. grandifolia (q), K. rotunda (r), K. candida (s), and Kaempferia sp 3 (t).

\section{DNA extraction, polymerase chain reaction, and DNA sequencing}

Genomic DNA was extracted from fresh young leaves or flowers of each plant using a modification of the CTAB method of Doyle and Doyle (1987). The psbA-trnH and petA-psbJ$p s b L$ regions of each taxon were separately amplified in a $50-\mu \mathrm{L}$ reaction volume containing $1 \mathrm{X}$ buffer, $\mathrm{MgCl}_{2}$ (1.5 and $3.0 \mathrm{mM}$ for $p s b A$-trnH and petA-psbJ-psbL, respectively), dNTPs $(0.20$ and $0.24 \mu \mathrm{M})$, primers $(0.20 \mu \mathrm{M}$ each of $p s b A-1 \mathrm{~F}$ : 5'-CTTGGTATGGAAGTAATGCA-3' and $t r n H-1 \mathrm{R}$ : 5'-ATCCACTTGGCTACATCCG-3', and $0.24 \mu \mathrm{M}$ each of petA-F: 5'-AGGTT CAATTGTMCGAAATG-3' and psbL-R: 5'-GTACTTGCTGTTTTATTTTC-3'), 200-400 ng total DNA and 1 U Taq DNA polymerase (Techaprasan et al., 2006). Polymerase chain reaction was carried out consisting of an initial denaturation at $94^{\circ} \mathrm{C}$ for 2 min followed by 35 cycles of denaturation at $94^{\circ} \mathrm{C}$ for $1 \mathrm{~min}$, annealing at $55^{\circ}$ or $58^{\circ} \mathrm{C}$ for $1 \mathrm{~min}$, and extension 
at $72^{\circ} \mathrm{C}$ for $30 \mathrm{~s}$ or $1 \mathrm{~min}$ for the respective regions. The final extension was carried out for $7 \mathrm{~min}$ at $72^{\circ} \mathrm{C}$. The amplified $p s b A-t r n H$ and petA-psbJ-psbL were direct-sequenced for both directions on an automated DNA sequencer using the original amplification primer as the sequencing primer.

\section{Data analysis}

Sequences of $p s b A$-trnH and petA-psbJ-psbL spacers were aligned using ClustalW incorporated in BioEdit version 7.0.5.2 (Hall, 1999) and further edited manually. Nucleotide sequence divergence between pairs of taxa was calculated using a Kimura (1980) 2-parameter model without indel consideration. Maximum parsimony analysis was carried out using Phylogenetic Analysis Using Parsimony (PAUP*) version 4.0 Beta 10 (Swofford, 2004). Gaps were treated as missing data. To reconstruct the most parsimonious trees, a heuristic search was executed using random (1000 replicates) stepwise addition followed by tree bisectionreconnection branch swapping. Bootstrapping (1000 replicates) was performed with random sequence addition. Bootstrapping values $(>50 \%)$ were superimposed on the strict consensus tree to illustrate confidence level of relationships among samples investigated in this study.

\section{RESULTS}

The amplified $p s b A$-trnH and petA-psbJ-psbL spacers in Kaempferia were approximately 800 and $1200 \mathrm{bp}$ in length with the exception of 400 and $900 \mathrm{bp}$ for $S$. biloba and 800 and 900 bp for S. minuta, respectively. Nucleotide sequences at the 3' end of petA-psbJ-psbL spacers were missing and not included in the analyses. Sequences of $p s b A-\operatorname{trn} H$ and partial petA-psbJ of all investigated specimens in this study were deposited in GenBank with accession Nos. GQ385976-386058 and GQ386059-386141, respectively.

Within Kaempferia, nucleotide sequences of psbA-trnH ranged between 714 bp ( $K$. elegans JT2007-8 and K. parviflora JT2007-17) and 798 bp (K. filifolia JT2007-23) and were $1010 \mathrm{bp}$ after multiple sequence alignments. Likewise, those of the partial petA-psbJ ranged between 659 bp (K. pulchra TT10144 and K. roscoeana TT16482) and 754 bp (K. rotunda TT15732) in length and resulted in the multiple alignments of $865 \mathrm{bp}$. For the combined data, $1875 \mathrm{bp}$ in length were obtained, including 126 variable parsimoniously uninformative sites and 116 parsimoniously informative characters (Table 2), indicating that both chloroplast DNA spacers provided limited phylogenetic information. Pairwise nucleotide divergence of psbA-trnH within Kaempferia and across all examined taxa ranged between 0.00-3.31 and $0.00-3.33 \%$ ( $K$. elegans TT16410 and G. thoreliana), while that of the partial petA-psbJ was between $0.00-2.03$ and $0.00-4.69 \%$ (K. fallax TT16670 and $S$. rubescens), respectively.

The strict consensus tree generated from the combined data of the spacers possessed 321 informative mutation steps with consistency and retention indices of 0.82 and 0.91 , respectively (Figure 3). Two accessions of $K$. candida were separated from other Kaempferia members. Disregarding K. candida, K. pulchra, and $K$. roscoeana, other Kaempferia species were separated into 4 major clades: clade A ( $K$. marginata, $K$. galanga, $K$. laotica, $K$. larsenii, $K$. albomaculata sp nov., $K$. pardi sp nov., and $K$. bambusetorum sp nov.); clade B includes only the Northeastern species $(K$. minuta sp nov., K. angustifolia, K. fallax, K. filifolia, and K. siamensis); clade C includes Kaempferia species whose inflorescences appear before leafy shoots, i.e., K. rotunda, $K$. grandifolia, Kaempferia sp 1, Kaempferia sp nov. 1, and Kaempferia sp nov. 2; clade D (K. elegans and K. parviflora). 
Table 2. Sequence characteristics and nucleotide sequence divergence of $p s b A$-trnH and petA-psbJ across Kaempferia species and outgroup references.

\begin{tabular}{|c|c|c|c|}
\hline & psbA-trnH $H^{\mathrm{a}}$ & petA-psbJ $J^{\mathrm{b}}$ & Combined data \\
\hline Number of nucleotides & 714-798 & $659-754$ & 1875 \\
\hline Number of uninformative-variable sites & 61 & 65 & 126 \\
\hline Number of informative characters & 68 & 48 & 116 \\
\hline Percentage of interspecific sequence divergence within Kaempferia ${ }^{\mathrm{c}}$ & $0.00-3.31$ & $0.00-2.03$ & $0.00-2.11$ \\
\hline Percentage of interspecific and intergeneric sequence divergence ${ }^{c}$ & $0.00-3.33$ & $0.00-4.69$ & $0.00-2.61$ \\
\hline \multicolumn{4}{|l|}{ Percentage of intraspecific sequence divergence } \\
\hline K. albomaculata sp nov. & 0.00 & 0.00 & 0.00 \\
\hline K. angustifolia & 0.00 & 0.00 & 0.00 \\
\hline K. bambusetorum $\mathrm{sp}$ nov. & 0.00 & 0.00 & 0.00 \\
\hline K. candida & 0.00 & 0.00 & 0.00 \\
\hline K. galanga & 0.00 & 0.00 & 0.00 \\
\hline K. laotica & 0.00 & 0.00 & 0.00 \\
\hline K. minuta sp nov. & 0.00 & 0.00 & 0.00 \\
\hline K. pardi sp nov. & 0.00 & 0.00 & 0.00 \\
\hline Kaempferia sp nov. 1 & 0.00 & 0.00 & 0.00 \\
\hline K. fallax & $0.00-0.27$ & $0.00-0.14$ & $0.00-0.14$ \\
\hline K. filifolia & 0.00 & $0.00-0.30$ & $0.00-0.14$ \\
\hline K. elegans & $0.00-0.98$ & $0.00-0.81$ & $0.00-0.77$ \\
\hline$K$. larsenii & 0.00 & $0.00-0.27$ & $0.00-0.14$ \\
\hline K. marginata & $0.00-0.41$ & $0.00-0.15$ & $0.00-0.28$ \\
\hline K. parviflora & $0.14-1.15$ & $0.45-0.76$ & $0.22-0.85$ \\
\hline K. pulchra & $0.00-0.83$ & $0.15-0.79$ & $0.07-0.59$ \\
\hline K. roscoeana & 0.00 & 0.61 & 0.29 \\
\hline K. rotunda & $0.00-0.82$ & $0.14-0.84$ & $0.14-0.83$ \\
\hline K. siamensis & 0.13 & 0.15 & 0.14 \\
\hline G. godefroyi & 0.56 & 0.70 & 0.63 \\
\hline G. thoreliana & 0.00 & 0.00 & 0.00 \\
\hline
\end{tabular}

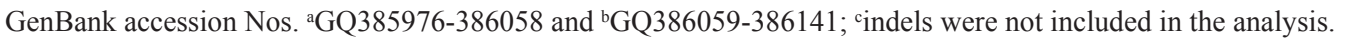

Like $K$. roscoeana, geographically different samples of Kaempferia species in clade B (except $K$. filifolia) clustered together. However, evolutionary relationships of other clades were not fully resolved, especially with $K$. elegans and $K$. parviflora, members of clade D. Within clade C, K. rotunda from different locations showed intraspecific sequence polymorphism and clustered separately with other taxa (Kaempferia sp 1, Kaempferia sp nov. 2, and K. grandifolia). However, two Kaempferia sp nov. 1 accessions grouped together ( $90 \%$ bootstrapping value). Members of clade A showed less informative sequence variation, resulting in unresolved evolutionary relationships. For example, K. galanga, K. marginata (except JT2007-4 and TT15721), and Kaempferia sp 4 possessed identical sequences. Moreover, only one indel could differentiate $K$. larsenii (except TT16540) and $K$. laotica.

No intraspecific sequence variation was observed in $K$. candida, $K$. angustifolia, $K$. laotica, $K$. galanga, $K$. pardi sp nov., $K$. bambusetorum sp nov., $K$. albomaculata sp nov., $K$. minuta sp nov., Kaempferia sp nov. 1, and G. thoreliana. In contrast, intraspecific sequence polymorphism was observed in different populations of $K$. fallax, $K$. filifolia, $K$. elegans, $K$. pulchra, K. rotunda, K. marginata, K. parviflora, K. larsenii, K. roscoeana, K. siamensis, and G. godefroyi.

Although phylogenetically unresolved relationships of some Kaempferia were observed, polymorphic sites and indels of $p s b A$-trnH and petA-psbJ can be used for species authentication of most Kaempferia species, i.e., K. laotica, K. angustifolia, $K$. siamensis, $K$. grandifolia, $K$. roscoeana, $K$. candida, $K$. pardi sp nov., $K$. bambusetorum sp nov., $K$. albomaculata sp nov., $K$. minuta sp nov., and Kaempferia sp nov. 1 and outgroups (Figures 4 and 5). Moreover, a string of sequences in $p s b A$-trnH (AGTTTAGATATTT) and petA-psbJ (CTACAA) could differentiate 
members of subgroup A1 (K. albomaculata sp nov./K. larsenii/K. laotica) and those of clade A from other taxa, respectively. In addition, species-specific sequences in $p s b A$-trn $H$ for $K$. albomaculata sp nov. (AGAT, G and TATATAAATATATAAA), $K$. bambusetorum (GTATATTAAATACAAAT) and K. laotica/K. larsenii (CATAA) were also found.

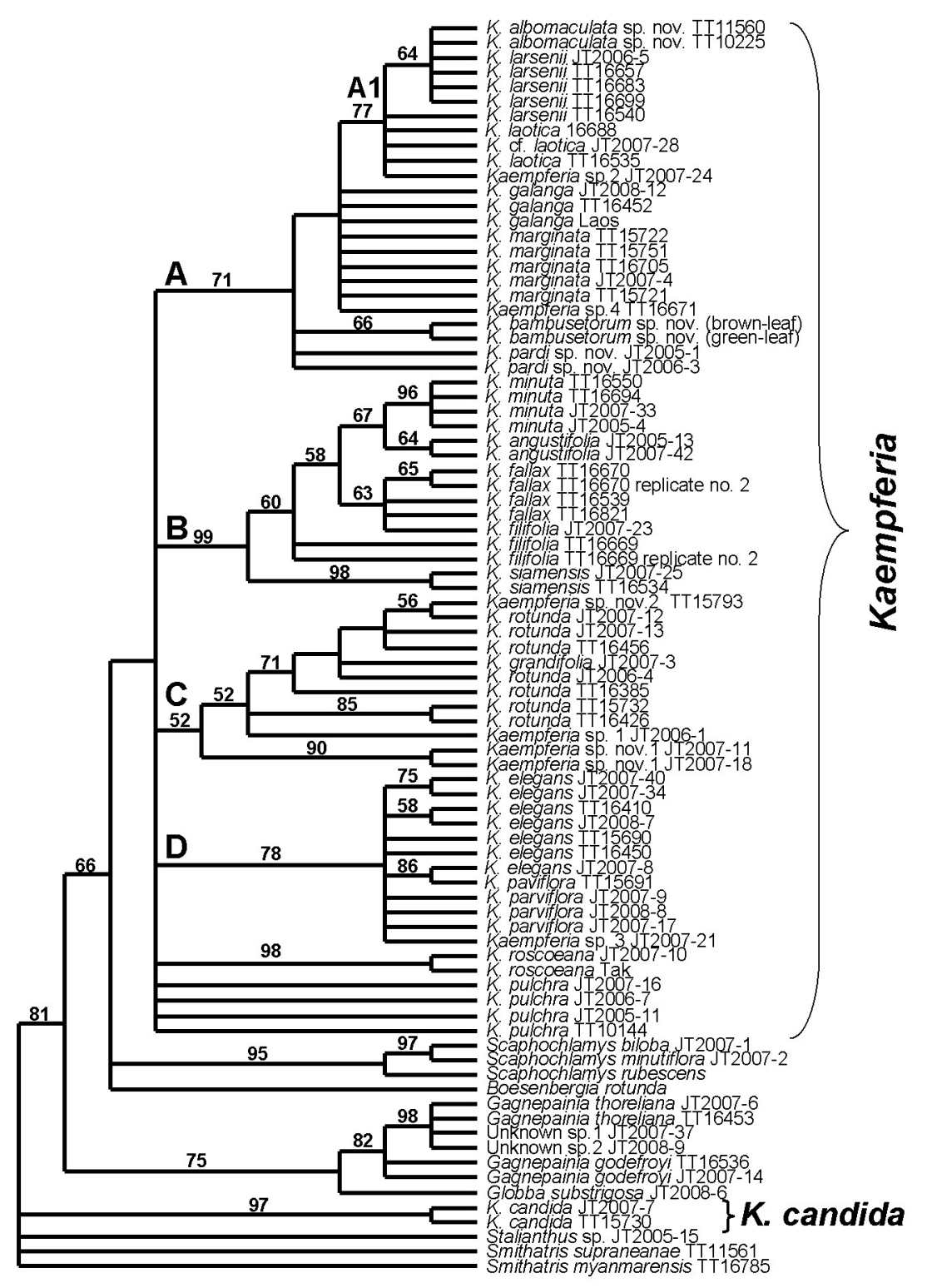

Figure 3. A strict consensus tree constructed from 778,100 of the most parsimonious trees based on combined $p s b A$-trn $H$ and $p e t A-p s b J$ sequences (tree length $=321$ steps; consistency index $=0.82$ and retention index $=0.91$ ). Values at the node $(>50 \%)$ represent the percentage of times that the particular node occurred in 1000 replicates generated by bootstrapping the original sequences. 


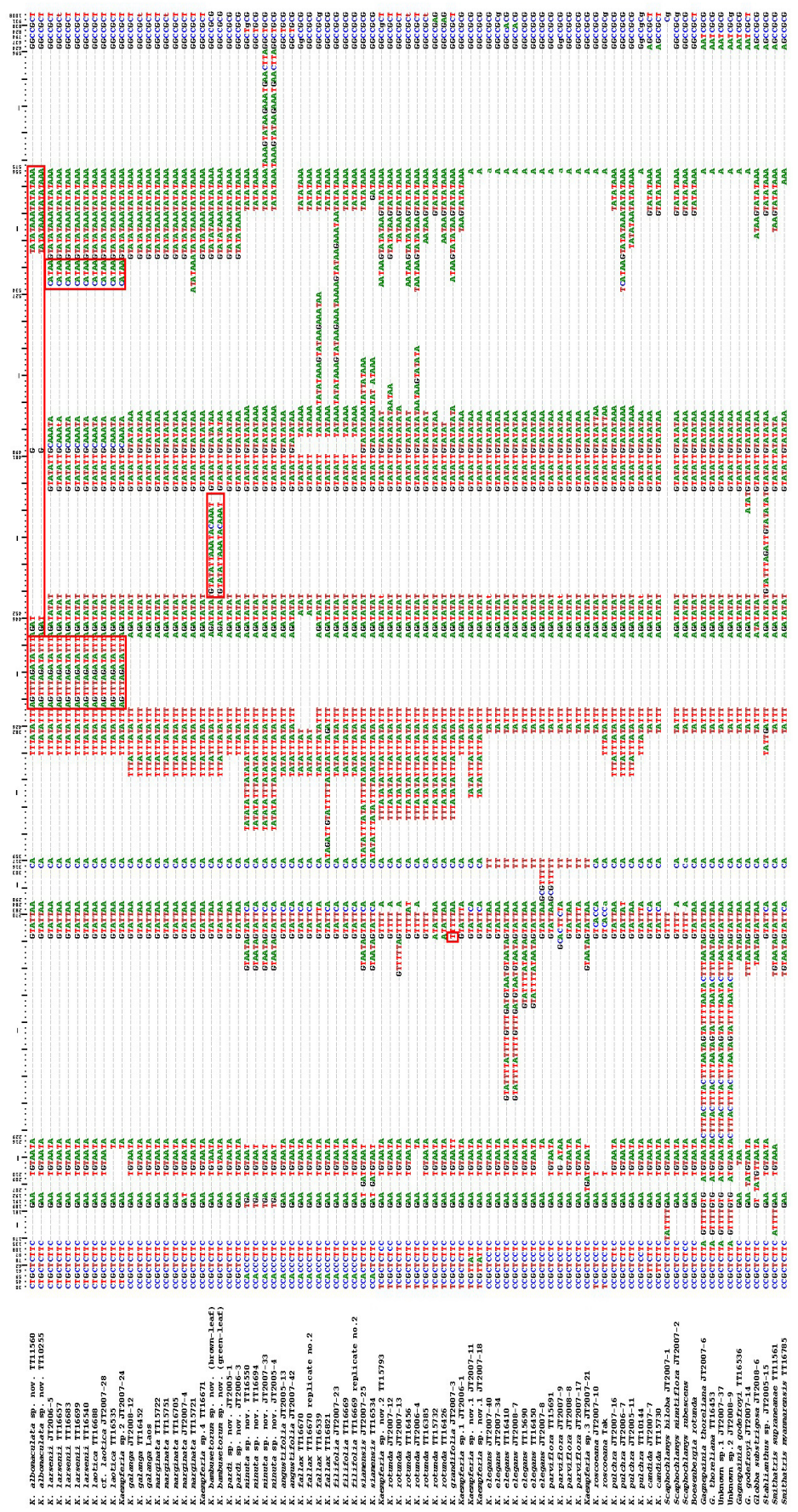

Figure 4. Polymorphic sites and indels of $p s b A-t r n H$ sequences used for authenticating most Kaempferia and outgroup species. Boxes indicate strings of sequences that are able to distinguish members of subgroup A1 and $K$. albomaculata, $K$. bambusetorum, K. laotica/K. larsenii, and $K$. grandifolia from other taxa. 


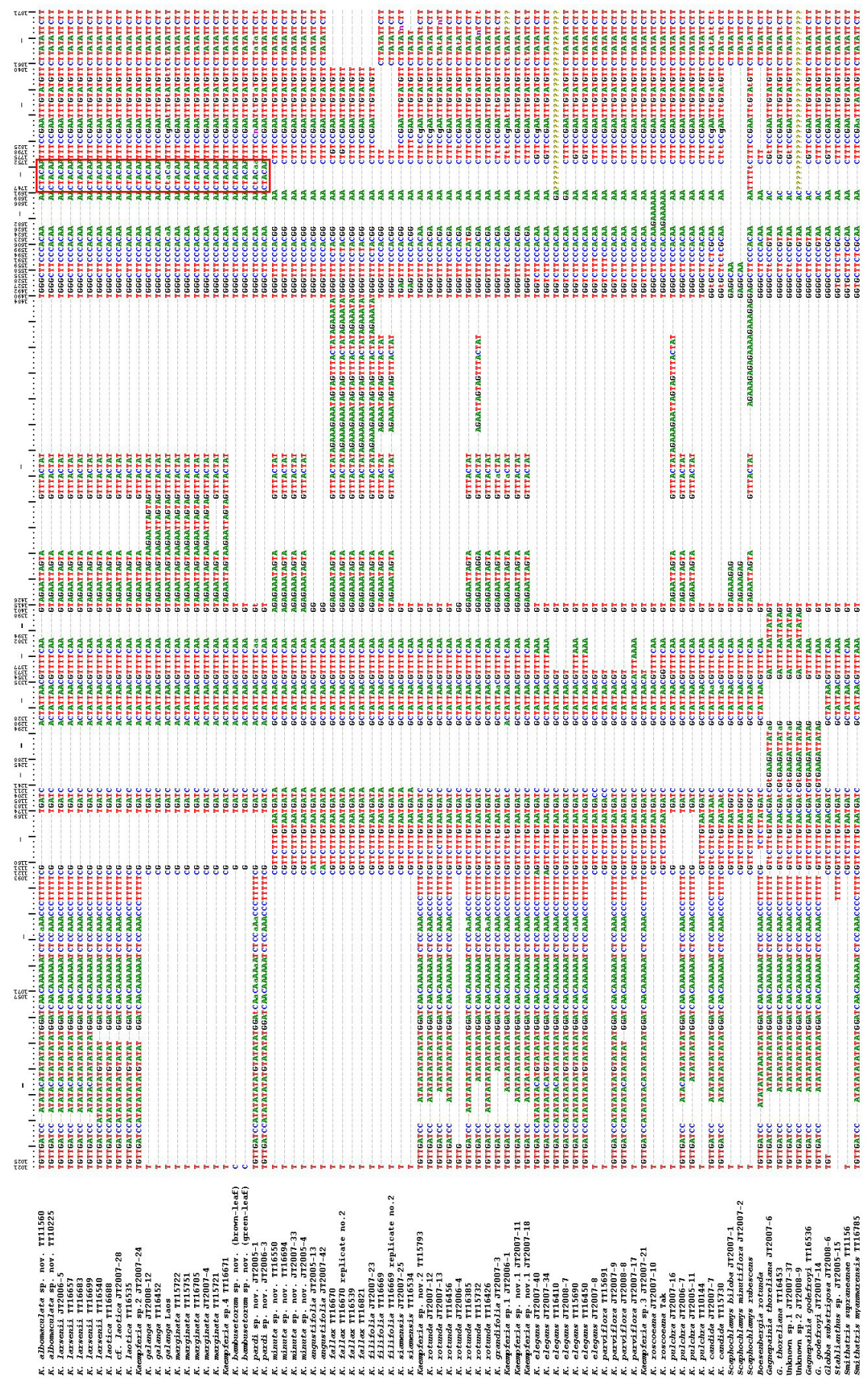

Figure 5. Polymorphic sites and indels of petA-psbJ sequences used for authenticating most Kaempferia and outgroup species. Boxes indicate strings of sequences that are able to distinguish members of clade A from other taxa. 


\section{DISCUSSION}

Like most genera (e.g., Globba, Curcuma, Alpinia, Boesenbergia, etc.) in Zingiberaceae, taxonomic difficulties were also observed in Kaempferia. In Boesenbergia, nucleotide polymorphisms and indels of $p s b A$-trn $H$ and petA-psbJ were successfully applied for species authentication, even though limited sequence divergence was observed (0.00-3.53 and 0.00-2.53\%, respectively; Techaprasan et al., 2006). Similarly, limited sequence divergence of $p s b A-t r n H$ and the partial petA-psbJ spacers in Kaempferia and outgroups (0.00-3.33 and $0.00-4.69 \%$, respectively) was also observed in this study.

Phylogenetic reconstruction based on the parsimony approach was carried out, but indels of the multiple aligned $p s b A-t r n H$ and petA-ps $b J$ sequences were excluded from the analysis as they caused low bootstrapping values in several other branches and more unresolved evolutionary relationships of the reconstructed tree (data not shown).

Previously, Kaempferia was regarded as a monophyletic genus (Kress et al., 2002). Based on our study on the maternally inherited chloroplast (cpDNA) data, K. candida was clearly allocated as the well-isolated clade outside Kaempferia members. The results suggest that $K$. candida may be misallocated to be a member of this genus. Nevertheless, we cannot rule out the possible consequence of reticulate evolution (e.g., intergeneric hybridization and introgression) in this species. Therefore, the taxonomic status of $K$. candida should be further examined by multiple loci of biparentally segregrated nuclear DNA markers to elucidate this speculation.

Phylogenetic analysis clearly revealed 4 different clades of Kaempferia in this study. Clade A consisted of complex species (e.g., K. marginata, K. galanga, and K. laotica). Of these, $K$. galanga is a cultivated species and believed to have been introduced from India (Holttum, 1950; Larsen and Saksuwan Larsen, 2006), whereas K. marginata is naturally distributed locally. Taxonomic key to Kaempferia species of Thailand described that leaf margin and labellum of $K$. marginata is purple, whereas leaf margin of $K$. galanga is usually white and its labellum is white with purple marking at the base (Sirirugsa, 1992). However, labellums of $K$. marginata display color variation from white to purple. Nucleotide sequences of psbA-trnH and petA-psbJ of K. galanga and K. marginata (excluding JT2007-4 and TT15721, which showed intraspecific polymorphism) were identical, suggesting that they are recently matriarchally diverse. More samples of $K$. marginata and $K$. galanga from the original resources (i.e., India) should be included in the analysis to elucidate whether $K$. galanga is a cultivated variant of $K$. marginata.

Within clade B, K. filifolia JT2007-23 clustered with K. fallax. Flowers of K. fallax and $K$. filifolia are similar but leaves of $K$. fallax are elliptic-linear to linear, whereas those of K. filifolia are filiform (Sirirugsa, 1992). Nucleotide polymorphism and indels of $p s b A$-trnH and petA-psbJ of $K$. filifolia from Pha Luang (TT16669 and TT16669 replicate No. 2) were obviously different from $K$. fallax from Sroi Sawan (TT16539), Pha Luang (TT16670 and TT16670 replicate No. 2), and Pha Chana Dai (TT16821), whereas those of K. filifolia from Pha Tam (JT2007-23) were similar to those of $K$. fallax. However, ITS sequences of these taxa are identical (data not shown). In addition, a phylogenetic tree inferred from amplified fragment length polymorphism (AFLP) analysis confirmed their close relationships (Techaprasan J, unpublished results). Accordingly, $K$. fallax and $K$. filifolia should be regarded as sibling species. K. siamensis, K. minuta sp nov. and $K$. angustifolia did not form a species complex and phylogenetically recognized as separate species. 
Loesener (1930) included three Kaempferia species in the subgenus Protanthium Horan. (K. rotunda, $K$. candida, and $K$. fissa) in the previous classification of Zingiberaceae. Based on cytological, anatomical and morphological studies, $K$. grandifolia was recently recognized as a new Kaempferia endemically found in Thailand. It exhibits the closest morphology to K. roscoeana and is classified as a member of the subgenus Soncorus Horan., but its inflorescences appear before leafy shoots, as seen in $K$. rotunda (Saensouk and Jenjittikul, 2001). In this study, $K$. grandifolia was phylogenetically placed between $K$. rotunda varieties. One polymorphic site ( $\mathrm{T}$ at position 266) in psbA-trnH could distinguish $K$. grandifolia from other species. Accordingly, we argue that $K$. grandifolia should be evolutionarily descended from the $K$. rotunda lineage.

There has been a controversy over the species status of $K$. elegans and $K$. pulchra (Holttum, 1950; Smith, 1987). Searle (1999) regarded K. pulchra Ridl. as a synonym of $K$. elegans Wall. However, several authors recognized these plants as different species (Sirirugsa, 1992; Larsen and Saksuwan Larsen, 2006). The anther crests of K. pulchra are clawedblade oblanceolate (leaf-shape) whereas those of K. elegans are orbicular (circular or nearly so; Sirirugsa, 1992). Our results revealed phylogenetic separation of $K$. pulchra from other Kaempferia species. Therefore, $K$. pulchra and $K$. elegans should be systematically recognized as different species.

The tree topology of Kaempferia in this study also indicated that K. parviflora and $K$. elegans were closely related phylogenetically, and 2 samples (K. parviflora TT15691 and $K$. elegans JT2007-8) may be their interspecific hybrid. More suitable molecular markers (e.g., polymorphic nuclear DNA and AFLP-derived markers) should be tested to identify whether introgression of cpDNA between $K$. parviflora TT15691 and K. elegans JT2007-8 as a consequence of hybridization readily occurs.

Generally, sequence polymorphism of $p s b A-t r n H$ and $p e t A-p s b J$ can be applied to DNA barcoding in Kaempferia. However, only one indel at the $5^{\prime}$ end of petA-psbJ was able to further differentiate $K$. laotica and $K$. larsenii (excluding TT16540). This indel seems to be a part of the inverted repeat of the stem-loop or hairpin structures commonly found in angiosperms (Kim and Lee, 2004), including Zingiberales (Swangpol et al., 2007). However, external morphology of leaves can be used to unambiguously differentiate $K$. larsenii and $K$. laotica. Leaves of $K$. larsenii are smaller $(0.5-1 \times 6-9 \mathrm{~cm})$, elliptic-linear and erect, whereas those of $K$. laotica are obviously larger (7-10.5 x 7-12.5 cm), suborbicular and horizontal close to the ground (Sirirugsa, 1992).

The 46-63-bp indels of the stem-loop structures at the $5^{\prime}$ end of petA-psbJ sequences were also observed in half of the Kaempferia investigated (e.g., K. laotica, K. larsenii, K. albomaculata sp nov., $K$. pardi sp nov., Kaempferia sp 1, Kaempferia sp nov. 1, Kaempferia sp nov. 2, some accessions of $K$. elegans, $K$. parviflora, $K$. pulchra, and $K$. rotunda) and 4 outgroups (B. rotunda, G. godefroyi, G. thoreliana, and S. myanmarensis) species. However, this DNA region may not be appropriate to either include in phylogenetic reconstruction or to use for DNA barcoding, owing to the possibility of being phylogenetically and systematically misleading (Kelchner and Wendel, 1996). For example, 6 accessions of $K$. elegans showed 4 different types of this indel, but it was absent in K. elegans JT2007-8.

Intraspecific sequence divergences in different populations of $K$. elegans $(0.00-0.77 \%), K$. parviflora $(0.22-0.85 \%), K$. pulchra $(0.07-0.59 \%)$, and $K$. rotunda $(0.14-0.83 \%)$ were greater than in other Kaempferia species indicating that these species were highly diverse. Therefore, $p s b A$-trnH and petA-psbJ spacers could not be used as DNA barcodes in K. elegans, K. parviflora, K. pulchra, K. ro- 
tunda, some K. larsenii, between K. marginata and K. galanga, and between $K$. fallax and $K$. filifolia .

Apart from that, polymorphic indels and nucleotides of psbA-trnH and petA-psbJ could be used for species authentication of most Kaempferia species (e.g., K. angustifolia, K. candida, K. laotica, K. roscoeana, K. siamensis, $K$. albomaculata sp nov., K. bambusetorum sp nov., K. minuta sp nov., K. pardi sp nov., and Kaempferia sp nov. 1) and outgroups (e.g., G. godefroyi and G. thoreliana) for which more than one specimen was available for each species.

Our results based on maternally inherited cpDNA data reveal that Kaempferia sp 1 should be recognized as newly unidentified Kaempferia species, and that Kaempferia sp 2, 3 , and 4 are not new species, exhibiting identical $p s b A-\operatorname{trn} H$ and petA-psbJ sequences to $K$. laotica, $K$. cf. parviflora, and the K. marginata/K. galanga species complex, respectively. Phylogenetic analysis also confirmed the species status of 4 newly described Kaempferia species (K. pardi sp nov., $K$. bambusetorum sp nov., $K$. albomaculata sp nov., and $K$. minuta sp nov.) and Kaempferia sp nov. 1. Although the floral parts of Kaempferia sp nov. 2 (TT15793) were clearly different from $K$. rotunda, nucleotide polymorphism and indels of $p s b A$-trnH and petA-psbJ were not sufficiently informative to verify that it is not a morphological variant of $K$. rotunda but a new Kaempferia species.

Apparently, psbA-trnH and petA-psbJ polymorphism unambiguously authenticated unknown species 1 and 2 as $G$. thoreliana. The inflorescence of the unknown sp 1 bloomed after collection for approximately one year, and it was concordantly classified as $G$. thoreliana on a morphological basis. Moreover, $p s b A$-trn $H$ and petA-psbJ sequences clearly suggest that the JT2007-28 specimen, which was initially misidentified as $K$. marginata, should be $K$. cf. laotica. This further confirms that $p s b A-t r n H$ and petA-psbJ spacers are potentially useful as DNA barcodes in most Kaempferia species found to be indigenous in Thailand.

Previously, Techaprasan et al. (2006) examined the sequence polymorphism of 22 Boesenbergia taxa at $3 \mathrm{cpDNA}$ regions ( $m a t K, p s b A-t r n H$, and petA-psbJ), and all taxa were unambiguously differentiated. In this study, polymorphism of psbA-trnH, and petA-psbJ in 71 Kaempferia and 14 outgroup taxa were examined. Informative characters of $p s b A$-trnH and $p e t A-p s b J$ sequences were sufficient to phylogenetically differentiate several taxa. Nucleotide polymorphism and indels of their sequences provided strong phylogenetic signals and could be applied in authenticating most Kaempferia and their closely related species. To confirm taxonomic status of problematic Kaempferia species (e.g., K. candida) and to clarify evolutionary relationships of this genus, a global sampling of Kaempferia and an examination by both biparentally inherited nuclear DNA (e.g., ribosomal ITS and polymorphic AFLP-derived sequences) and these ( $p s b A-t r n H$ and petA-psbJ) or other cpDNA regions are required for studying the molecular systematics of the whole genus Kaempferia.

\section{ACKNOWLEDGMENTS}

The authors would like to acknowledge Ms. Sirikan Prasertluk and Ms. Parichat Chumthong for their laboratory assistance and thank Dr. Jennifer Luangsa-ard, Mr. Poonsak Vacharakorn, Ms. Satinee Suetrong, and Ms. Wiwantanee Chaygate for providing some samples. We also thank Dr. Jennifer Luangsa-ard for useful comments on the manuscript. Research supported by the Thailand Research Funds (TRF) and the National Center for Genetic Engineering and Biotechnology (BIOTEC) for Biodiversity Research and Training Program (BRT) (grants \#BRT R250001 and \#R251137). 


\section{REFERENCES}

Chuakul W (2003). Ethnomedical Uses of Thai Zingiberaceous Plants. In: Proceedings of the 3rd International Symposium on the Family Zingiberaceae (Chantaranothai P, Larsen K, Sirirugsa P and Simpson D, eds.). Applied Taxonomic Research Center, Khon Kaen University, Khon Kaen, July 7-12, 2002, 201-207.

Doyle JJ and Doyle JL (1987). A rapid DNA isolation procedure for small quantities of fresh leaf tissue. Phytoch. Bull. 19: 11-15.

Hall TA (1999). BioEdit: a user-friendly biological sequence alignment editor and analysis program for Windows 95/98/NT. Nucl. Acids Symp. Ser. 41: 95-98. Available at [http://www.mbio.ncsu.edu/BioEdit/bioedit.html]. Accessed July 25, 2008.

Holttum RE (1950). The Zingiberaceae of the Malay Peninsula. Gard. Bull. Singap. 13: 117-123.

Ibrahim H (1999). Kaempferia. In: Plant Resources of South-East Asia (de Padua LS, Bunyapraphatsara N and Lemmens RHMJ, eds.). Vol. 12. Backhuys Publisher, Leiden, 331-335.

Jenjittikul T and Larsen K (2000). Kaempferia candida Wall. (Zingiberaceae), a new record for Thailand. Thai For. Bull. 28: 45-49.

Kelchner SA and Wendel JF (1996). Hairpins create minute inversions in non-coding regions of chloroplast DNA. Curr. Genet. 30: 259-262.

Kim KJ and Lee HL (2004). Complete chloroplast genome sequences from Korean ginseng (Panax schinseng Nees) and comparative analysis of sequence evolution among 17 vascular plants. DNA Res. 11: 247-261.

Kimura M (1980). A simple method for estimating evolutionary rates of base substitutions through comparative studies of nucleotide sequences. J. Mol. Evol. 16: 111-120.

Kress WJ, Prince LM and Williams KJ (2002). The phylogeny and a new classification of the gingers (Zingiberaceae): evidence from molecular data. Am. J. Bot. 89: 1682-1696.

Kress WJ, Liu AZ, Newman M and Li QJ (2005a). The molecular phylogeny of Alpinia (Zingiberaceae): a complex and polyphyletic genus of gingers. Am. J. Bot. 92: 167-178.

Kress WJ, Wurdack KJ, Zimmer EA, Weigt LA, et al. (2005b). Use of DNA barcodes to identify flowering plants. Proc. Natl. Acad. Sci. U. S. A. 102: 8369-8374.

Larsen K and Saksuwan Larsen S (2006). Gingers of Thailand. Queen Sirikit Botanic Garden. The Botanical Garden Organization, Chiang Mai.

Loesener T (1930). Zingiberaceae. In: Die Natürlichen Pflanzenfamilien (Engler A and Prantl K, eds.). W. Engelmann, Leipzig, 541-640.

Saensouk S and Jenjittikul T (2001). Kaempferia grandifolia sp. nov. (Zingiberaceae) a new species from Thailand. Nord. J. Bot. 21: 139-142.

Searle RJ (1999). A new combination and new synonymy in Kaempferia (Zingiberaceae: Hedychieae). Telopea 8: $375-376$.

Sirirugsa P (1992). Taxonomy of the genus Kaempferia (Zingiberaceae) in Thailand. Thai For. Bull. 19: 1-15.

Smith RM (1987). A review of Bornean Zingiberaceae: III (Hedychieae). Notes R. Bot. Gard. Edinb. 44: 203-232.

Sookkongwaree K, Geitmann M, Roengsumran S, Petsom A, et al. (2006). Inhibition of viral proteases by Zingiberaceae extracts and flavonoids isolated from Kaempferia parviflora. Pharmazie 61: 717-721.

Soontornchainaksaeng P (2005). Reports on Chromosome Numbers of Plants in Thailand. BRT program. Chuan Printing Press Ltd., Part. Bangkok.

Swangpol S, Volkaert H, Sotto RC and Seelanan T (2007). Utility of selected non-coding chloroplast DNA sequences for lineage assessment of Musa interspecific hybrids. J. Biochem. Mol. Biol. 40: 577-587.

Swofford DL (2004). PAUP*: Phylogenetic Analysis Using Parsimony (* and Other Methods). Version 4.0 Beta 10. Sinauer Associates, Sunderland.

Techaprasan J, Ngamriabsakul C, Klinbunga S, Chusacultanachai S, et al. (2006). Genetic variation and species identification of Thai Boesenbergia (Zingiberaceae) analyzed by chloroplast DNA polymorphism. J. Biochem. Mol. Biol. 39: 361-370.

Thongnest S, Mahidol C, Sutthivaiyakit S and Ruchirawat S (2005). Oxygenated pimarane diterpenes from Kaempferia marginata. J. Nat. Prod. 68: 1632-1636.

Vimala S, Norhanom AW and Yadav M (1999). Anti-tumour promoter activity in Malaysian ginger rhizobia used in traditional medicine. Br. J. Cancer 80: 110-116.

Yenjai C, Daodee S and Wangboonskul J (2003a). Antifungal Activity and Antimycobacterial Activity of Ethyl p-methoxycinnamate from Kaempferia galanga L. In: Proceedings of the 3rd International Symposium on the Family Zingiberaceae (Chantaranothai P, Larsen K, Sirirugsa P and Simpson D, eds.). Applied Taxonomic 
Research Center, Khon Kaen University, Khon Kaen, July 7-12, 2002, 193-195.

Yenjai C, Kittakoop P, Daodee S and Prasanphen K (2003b). Antimycobacterial and Antifungal Activities of Flavonoids from Kaempferia parviflora Wall. ex Bak. In: Proceedings of the 3rd International Symposium on the Family Zingiberaceae (Chantaranothai P, Larsen K, Sirirugsa P and Simpson D, eds.). Applied Taxonomic Research Center, Khon Kaen University, Khon Kaen, July 7-12, 2002, 190-192.

Yenjai C, Prasanphen K, Daodee S, Wongpanich V, et al. (2004). Bioactive flavonoids from Kaempferia parviflora. Fitoterapia 75: 89-92. 